\title{
A trajetória persistente do Boletim Epidemiológico Paulista - Bepa
}

\author{
Boletim Epidemiológico Paulista (Paulista Epidemiologic Bulletin) \\ - persistent trajectory
}

Clelia Maria Sarmento de Souza Aranda ${ }^{1}$

Lilian Nunes Schiavon ${ }^{2}$

Sylia Rehder ${ }^{3}$

${ }^{1}$ Coordenadoria de Controle de Doenças,

Secretaria de Estado da Saúde de São Paulo (SES-

SP). Av. Dr. Arnaldo $351 / 1^{\circ}$,

Cerqueira Cezar. 01246-000

São Paulo SP Brasil.

bepa@saude.sp.gov.br

${ }^{2}$ Centro Técnico

de Documentação,

Coordenação das

Bibliotecas, Coordenadoria

de Controle de Doenças

(CCD), SES-SP.

${ }^{3}$ Centro de Produção e

Divulgação Científica, CCD,

SES-SP.

\begin{abstract}
The Paulista Epidemiologic Bulletin (Bepa), created in 2004 is a monthly publication from the Coordinating Office of Diseases Control (CCD), from the State Office of Health of Sao Paulo (SES-SP) responsible for planning and executing actions of health promotion and preventing any risks and diseases throughout the covered areas of the system (SUS-SP). Originally as a proposal of a fast publication of objective informations it suffered transformations along the years and turned out to be an important tool for service actions and health managers. Funded exclusively by public resources it tries hard to maintain the monthly periodicity and disclosure of epidemiologic data, description of programmatic actions, health situation analysis and striking reports concerning the history of public health in Sao Paulo.
\end{abstract}

Key words Periodic, Communication and Scientific divulgation, Spread of information
Resumo O Bepa-Boletim Epidemiológico Paulista, criado em 2004, é uma publicação mensal da Coordenadoria de Controle de Doenças (CCD), órgão da Secretaria de Estado da Saúde de São Paulo (SES-SP) responsável pelo planejamento e execução das ações de promoção à saúde e prevenção de quaisquer riscos, agravos e doenças, nas diversas áreas de abrangência do Sistema Único de Saúde de São Paulo (SUS-SP). Originalmente tendo como proposta a publicação ágil de informações objetivas, sofreu transformações ao longo dos anos e configurou-se como ferramenta para ações do serviço e gestores de saúde. Financiado exclusivamente com recursos públicos, esforça-se para manter a periodicidade mensal e a divulgação de dados epidemiológicos, descrição de ações programáticas, análises de situação de saúde e relatos marcantes para a história da saúde pública paulista.

Palavras-chave Periódico, Comunicação e Divulgação cientifica, Disseminação de informação 


\section{Introdução}

O Boletim Epidemiológico Paulista (Bepa), criado em 2004, é uma publicação mensal da Coordenadoria de Controle de Doenças (CCD), órgão da Secretaria de Estado da Saúde de São Paulo (SES-SP) responsável pelo planejamento e execução das ações de promoção à saúde e prevenção de quaisquer riscos, agravos e doenças, nas diversas áreas de abrangência do Sistema Único de Saúde de São Paulo (SUS-SP).

Idealizado pelo Prof Dr Luiz Jacintho da Silva, apontava em sua primeira edição ${ }^{1}-$ de janeiro de 2004 - a proposta de configurar-se como boletim, a exemplo do Morbidity and Mortality Weekly Report (MMWR) do Centers for Disease Controle and Prevention e do Eurosurveillance Weekly da União Europeia, com periodicidade mensal e disponibilização eletrônica. O conteúdo vislumbrava publicar de forma ágil informações objetivas sobre doenças e agravos de interesse de saúde púbica, que tinham sido objeto de estudo ou ação em período recente no estado de São Paulo.

Nessa trajetória de 11 anos (atualmente na sua 134 a edição), o Bepa foi sofrendo transformações e adequações. Uma delas atribuiu à publicação um caráter mais científico, com a introdução de regras para a publicação e revisão de artigos por pares².

A coordenação editorial adotou como regramento inserir pelo menos um artigo original em cada edição, seguido de outras categorias de artigos como de atualização, opinião ou especiais, sempre sobre temas relevantes da saúde pública paulista. Além disso, o Bepa se constituiu em importante veículo para divulgação de normas e informes técnicos detalhados das ações programáticas de Vigilância em Saúde. Edições especiais são também produzidas proporcionando registro de marcos histórico da Saúde Pública Paulista.

\section{Histórico}

Em 2003, na Secretaria de Estado da Saúde, ocorria o reordenamento da estrutura da vigilância em saúde paulista sob o nome de Agência Paulista de Controle de Doenças, instituição que agregaria os departamentos e institutos da Secretaria de Estado da Saúde responsáveis pelas ações de vigilância em saúde, completando um ciclo centenário iniciado pela instituição do Serviço Sanitário de São Paulo, no século XIX.

Coordenando esse processo estava o médico especialista em medicina preventiva, professor da Unicamp e ex-superintendente da Sucen (Superintendência de Controle de Endemias), Luiz Ja- cintho da Silva. Integração e articulação entre as diversas instituições da antiga CIP (Coordenadoria dos Institutos de Pesquisa) - embrião do que seria a atual $\mathrm{CCD}^{3,4}$ (Coordenadoria de Controle de Doenças) - eram algumas das palavras de ordem nesse movimento, do qual faziam parte: Centro de Vigilância Epidemiológica, Centro de Vigilância Sanitária, Instituto Adolfo Lutz, Instituto Pasteur, Centro de Referência e Treinamento em DST/Aids-SP, Instituto Lauro de Souza Lima e Instituto Clemente Ferreira.

A comunicação foi rapidamente identificada como um importante pilar desse processo. Visionário, Luiz Jacintho demandou a criação de uma revista que divulgasse de forma ágil as questões relevantes para a saúde pública e que contribuísse para uma maior interface entre as instituições.

Como legítimo pai da ideia, Luiz Jacintho orientou para que o projeto editorial fosse inspirado no MMWR, do Centers for Disease Control and Prevention (CDC/EUA), e completou dando o nome de Boletim Epidemiológico Paulista, cuja sigla Bepa lhe pareceu simpática e apropriada. "A pronúncia parece até uma palavra italiana", comentava bem humorado enquanto fazia a divulgação da revista nos diversos fóruns dos quais participou. Nascia assim o Bepa, sob a assinatura autoral de seu primeiro editor.

Proposta ousada para uma revista institucional de caráter técnico científico, o Bepa adotou a periodicidade mensal, sem dúvida um diferencial que tem sido respeitado ao longo desses anos, fruto da perseverança da equipe de coordenadores editoriais.

Os diversos editores que sucederam Luiz Jacintho também imprimiram a marca da sua condução na revista, seja em editoriais, seja na indicação sobre qual a melhor forma da publicação atingir seu objetivo. Nomes entre os mais brilhantes sanitaristas e infectologistas, que também fizeram diferença na gestão da vigilância em saúde paulista à frente da CCD: Marcos Boulos (atual); Clelia Aranda ( 2007 a 2011) e Carlos Magno Fortaleza (2005 a 2006).

\section{Uma publicação para gestores e profissionais de saúde}

Se no início o Bepa era veiculado em versão eletrônica, já nas primeiras edições foi identificada a necessidade de existir também uma versão impressa, principalmente para constituir acervo de consulta para seu público alvo formado por gestores e profissionais de saúde. Atualmente o Bepa circula um mil exemplares impressos, composto cada edição pelo conteúdo de duas edições ele- 
trônicas, totalizando seis edições impressas ao ano (Figura 1).

A sua produção está sob a responsabilidade do Centro de Produção e Divulgação Cientifica da CCD, equipe multiprofissional da área de comunicação, com apenas quatro membros, coordenada por uma jornalista. Além da produção do Bepa, o Centro viabiliza a elaboração de material de apoio aos eventos da instituição e mantém canal permanente com o Gabinete do Secretário para divulgação da informação cientifica em veículos de comunicação de massa.

O periódico divulga dados epidemiológicos, descrição de ações programáticas, análises de situação de saúde ${ }^{5}$ e documenta relatos marcantes para a história da saúde pública paulista como: implementação da vigilância clinicolaboratorial da influenza nos serviços públicos, inicio das campanhas de vacinação contra influenza, atuação da primeira turma de alunos do EPI-SUS (curso de formação em epidemiologia de campo), identificação de agravos não transmissíveis como problema de saúde pública (violência sexual, acidentes automobilísticos, homicídios e moradores de rua), casos de febre Maculosa na região Metropolitana da Capital, investigação epidemiológica para implantes mamários.

Também merece destaque a inserção de temas associados à atuação da Vigilância Sanitária e Ambiental como análise fiscal de alimentos, vigilância da qualidade da água para consumo humano e investigação epidemiológica para exposição ao chumbo.

Dentre a tipologia de artigos, a publicação de resumos de dissertações e teses, até dois anos da defesa, tem propiciado apoio na divulgação de pesquisas realizadas no Programa de Pós Graduação em Ciências da CCD. Recomendado pelo Conselho Técnico Científico da Fundação Coordenação de Aperfeiçoamento de Pessoal de Nível Superior (CAPES) e reconhecido pelo Conselho Nacional de Educação desde 2000, para os níveis de mestrado e doutorado, o Programa tem forte atuação voltada para o serviço e envolve a experiência acumulada pelos pesquisadores das instituições centenárias da Secretaria de Saúde.

O Bepa se consolidou como ferramenta de informação em saúde e passou a pautar os veículos de comunicação. Algumas de suas edições tiveram intensa repercussão na mídia, despertando discussões e polêmicas.

\section{Bepa registra fatos da saúde pública e integra a Rede de Informação e Conhecimento (RIC/SES)}

Na sua edição comemorativa do centésimo número (2012), o Bepa reúne artigos escritos pelos quatros coordenadores da CCD, também editores da publicação ${ }^{6}$. Temas importantes da história recente da saúde pública e do seu futuro ficaram registrados nessa edição: o acompanhamento de perto da primeira pandemia do século XXI; difusão da vigilância sobre infecções hospitalares; um olhar para o futuro mostrando as perspectivas de uma vacina contra a dengue e o cenário das doenças num mundo globalizado.

Edições temáticas também compõem o acervo do Bepa, a exemplo da recente edição novembro/dezembro de 2014 registrando a certificação de "Eliminação do T.infestans como problema de Saúde Pública no Estado de São Paulo”, conferido pela Comissão Intergovernamental da Iniciativa Sub-regional do Cone Sul/Organização Pan-Americana de Saúde ${ }^{7}$.

A versão eletrônica alcança inúmeras instituições como outras secretarias de saúde estaduais e os mais diversos serviços de saúde. Disponível no Portal de Revistas da Secretaria de Estado da Saúde de São Paulo, iniciativa em parceria com o Centro Latino-Americano e do Caribe de Informação em Ciências da Saúde (Bireme), utiliza o modelo SciELO de publicação eletrônica.

O Portal, integrante da Biblioteca Virtual de Saúde/ Rede de Informação e Conhecimento,

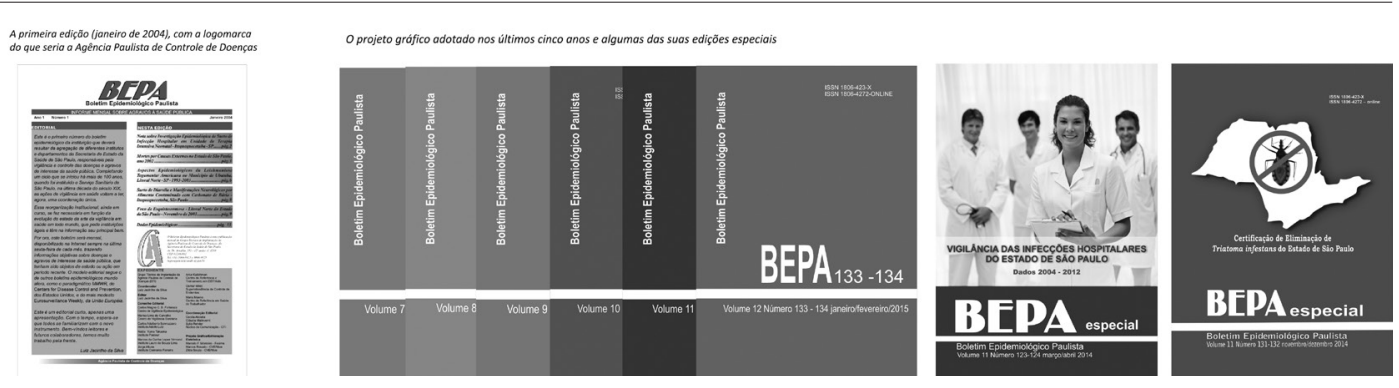

Figura 1. Edições do Boletim Epidemiológico Paulista (BEPA), 2004 a 2011. 
http://ses.sp.bvs.br, foi construído para organizar e unificar a publicação das revistas produzidas no âmbito da Secretaria, até então dispersos em diferentes sítios e formatos, com o intuito de ampliar sua visibilidade. Para integrar inicialmente este Portal, após a realização de um diagnóstico, foram selecionados os periódicos da pasta melhor estruturados, entre eles o Boletim Epidemiológico Paulista, e condizentes com os critérios estabelecidos. O processo de implantação contou com várias etapas, incluindo a conscientização dos editores quanto às necessidades de algumas mudanças no processo de publicação, a capacitação de equipe para a marcação e conversão de artigos e a realização de um Workshop direcionado aos editores, visando permitir maior detalhamento sobre os benefícios e as possibilidades do novo Portal. Estão disponíveis no Portal de Revistas Saúde SP outros quatro títulos: Boletim do Instituto de Saúde, Revista do Adolfo Lutz, Cadernos de História da Ciência e Hansenologia Internationalis. Dentre os 1.600 artigos, 364 são do Bepa.

Oitenta e quatro edições do Bepa (62\% do acervo), janeiro/2007 a novembro/2013, encontram-se com metodologia de acesso unificada (SciELO), totalizando mais de 52,8 mil acessos aos seus artigos. Esforços são empreendidos para ampliar e atualizar a disponibilização, o que nem sempre é rápido ou fácil para iniciativas com recursos, humanos e financeiros, exclusivamente públicos.

No entanto, as demais edições mantêm-se no sitio eletrônico da CCD conferindo ao Boletim a característica marcante de constituir ferramenta para ações do serviço e apoio a gestores de saúde. A revista também integra o conteúdo de materiais didáticos da rede estadual de educação e é indicada como bibliografia em concursos públicos.

\section{Considerações finais}

O Bepa é hoje uma das mais longevas revistas no segmento. O compromisso com a periodicidade mensal e com a do vigor editorial são frutos do trabalho dedicado da sua equipe à frente da coordenação editorial.

A revista optou por manter seu caráter endógeno, garantindo o espaço para temas de interesse da gestão paulista, mas garante a publicação de artigos e informes de outros estados e países, sempre que forem relevantes para a saúde pública.

O esforço para os próximos anos é conseguir diminuir cada vez mais o tempo entre a ocorrência do fato ou da produção científica e sua publicação. O Bepa pretende com isso contribuir para que seu leitor tenha a informação precisa e em tempo oportuno, que auxilie no seu trabalho, análise e tomada de decisão.

\section{Colaboradores}

CMSS Aranda, LN Schiavon e S Rehder participaram igualmente de todas as etapas de elaboração do artigo.

\section{Referências}

1. Boletim Epidemiológico Paulista (Bepa). Bepa 2004; 1(1). [acessado 2015 abr 15]. Disponível em: http:// www.saude.sp.gov.br/resources/ccd/homepage/bepa 12004/1_de_2004.pdf

2. Boletim Epidemiológico Paulista (Bepa). Instruções aos autores. Bepa 2013; 10(109):28-31. [acessado 2015 abr 15]. Disponível em: http://www.saude.sp.gov.br/ resources/ccd/homepage/bepa/instrucoes_2013_nova. pdf

3. São Paulo (Estado). Decreto no 49.343 de 24 de janeiro de 2005. Dispõe sobre as Coordenadorias da Secretaria da Saúde. Diário Oficial do Estado de São Paulo 2005; 25 jan.

4. São Paulo (Estado). Decreto no 54.739, de 2 de setembro de 2009. Reorganiza a Coordenadoria de Controle de Doenças - CCD, da Secretaria da Saúde, e dá providências correlatas. Diário Oficial do Estado de São Paulo 2009; 03 set.

5. Boletim Epidemiológico Paulista (Bepa). Bepa 2012; 9(Especial). [acessado 2015 abr 15]. Disponível em: http://www.saude.sp.gov.br/resources/ccd/homepage/ bepa/2012/edicao_especial.pdf
6. Boletim Epidemiológico Paulista (Bepa). Certificação de eliminação de Triatoma infestans do Estado de São Paulo. Bepa 2014; 11(Esp):131-132. [acessado 2015 abr 15]. Disponível em: http://www.saude.sp.gov.br/ resources/ccd/homepage/bepa/edicao-2014/edicao _131-132_-_novembro_dezembro.pdf

7. Boletim eletrônico GAIS Informa [homepage na internet]. São Paulo. Secretaria de Estado da Saúde. Coordenadoria de Planejamento em Saúde. Grupo Técnico de Avaliação e Informação em Saúde. [acessado 2015 abr 15]. Disponível em: http://portal.saude.sp.gov.br/ ses/perfil/profissional-da-saude/gais-informa/boletimeletronico-gais-informa

Artigo apresentado em 28/04/2015

Aprovado em 05/05/202015

Versão final apresentada em 07/05/2015 